\title{
Impact and Trends in Global Ophthalmology
}

\section{Lloyd B. Williams ${ }^{1,2} \cdot$ S. Grace Prakalapakorn ${ }^{1,2,3} \cdot$ Zubair Ansari $^{4} \cdot$ Raquel Goldhardt $^{4}$}

Published online: 22 June 2020

(C) Springer Science+Business Media, LLC, part of Springer Nature 2020

\begin{abstract}
Purpose of Review Our goal is to provide a review of the impact, global estimates, and projection of vision impairment as well as ongoing systems for eye care delivery.

Recent Findings Many of the blinding diseases in developing countries are preventable or curable, but the lack of ophthalmologists, the lack of education, and the lack of access to any eye care are some of the major obstacles encountered.

Summary As our world becomes more interconnected through globalization, the interactions between different cultures and populations increase. Global ophthalmology is a field dedicated to building sustainable eye care delivery systems to deliver highquality care in minimal resource settings, with the aim of reducing blindness around the world.
\end{abstract}

Keywords Global ophthalmology · World Health Organization · Cataract · Diabetic retinopathy · Trachoma · Glaucoma • Coronavirus

\section{Introduction}

On October 8, 2019, the World Health Organization (WHO) released a World Report on vision that stated there are over 2.2 billion people with vision impairment or blindness worldwide, and over 1 billion of those have conditions that could be treated or prevented [1]. Globally, the burden of vision impairment and blindness is greatest in low- and middle-income countries. Despite effective and relatively low-cost interventions, refractive error and cataract remain the largest causes of vision impairment and blindness. There is expected to be an increasing demand for global eye care due to an aging population, changing lifestyles (leading to increased lifestyle-related blindness (e.g., diabetes)), and population growth. The global eye health report indicates several challenges in meeting this anticipated

This article is part of the Topical Collection on Retina

Lloyd B. Williams and S. Grace Prakalapakorn are co-first authors.

Raquel Goldhardt

rgoldhardt@med.miami.edu

1 Department of Ophthalmology, Duke University, Durham, NC, USA

2 Duke Global Health Institute, Duke University, Durham, NC, USA

3 Department of Pediatrics, Duke University, Durham, NC, USA

4 Department of Ophthalmology, Bascom Palmer Eye Institute, University of Miami Miller School of Medicine, 900 NW 17th St, Office \#275, Miami, FL 33136, USA demand, including poor data to aid in planning and lack of integration of eye care into overall healthcare systems.

The WHO Global Action Plan emphasizes a delivery model relying on primary eye care as an approach to tackle visual impairment. One recently reported example of primary eye care enhancement is the Global Sight Initiative [2]. The Global Sight Initiative is a strategic initiative of the Seva Foundation and supports a collection of non-governmental organizations and eye hospitals collaborating to reduce blindness and increase services. Vision centers in this model aim to accomplish the following: (1) provide primary eye care so referral hospitals can divert their resources to focus on sightsaving surgical interventions, (2) identify patients that need surgical intervention or urgent referral for sight-saving treatment, (3) help patients and families overcome barriers (fear, cost, transportation, lack of awareness of eye care needs) to receive advanced medical or surgical care, (4) reduce cost to patients by decreasing geographic barriers, (5) improve healthcare-seeking behavior and follow-up, (6) improve gender equity of care, as vision centers have been shown to improve access for women by reducing cultural and transportation barriers, and (7) improve community involvement, which provides economic development for the community and better compliance [2]. The vision centers carry out three primary functions-recognize eye conditions, refract and provide spectacles, and refer patients to the sponsoring institution as needed. They also provide low vision and school and community eye services. 
Progress in eye care can and is being made. A recent example is a collaboration between Sightsavers and the government of Nigeria. A Vision 2020 Right to Sight strategy was implemented between 2005 and 2014 in Sokoto State, Nigeria. The zone chosen for the intervention had the lowest eye health indices in a 2005 baseline survey. Over the decade, the number of ophthalmologists increased from zero to six and cataract surgery coverage from 7.1 to $62 \%$, and among people over 50 years old, visual impairment decreased from 45.3 to $22.7 \%$ [3].

LV Prasad Eye Institute (based in Hyderabad, India) created a system which is testing application of its eye care delivery system in Liberia, West Africa. The success of cross-cultural application of processes is of particular importance to developing eye care in countries that have the least resources. LV Prasad uses a graded fee structure for the same clinical care, but provides the option of additional non-clinical services for a higher fee, i.e., air-conditioned waiting room, dedicated appointments, and more. LV Prasad also uses a clinical urgency-based 5-tiered system ranging from the quaternary referral center to basic rural eye care and screening [4]. This review presents details about the LV Prasad system and we await the results of this endeavor in Liberia.

\section{Cataract}

Cataract is the most common cause of vision impairment or blindness, affecting 65 million people worldwide [1]. Numerous ongoing public efforts have been devised to tackle the global cataract burden, but in recent years, these efforts have been focused on the often variable outcomes of surgery. In Malaysia, a cumulative sum analysis metric was introduced in 2009 to assess the quality of cataract surgery outcomes. They evaluated 1300 trainees and surgeons who together perform $50-70 \%$ of cataract surgeries nationwide. Surgeons who fell below acceptable levels were mentored and monitored for improvement. Over the implementation period from 2007 to 2017, posterior capsule breakage rates fell from 4.2 to $2.4 \%$ and post-operative best spectacle-corrected visual acuity (BSCVA) > 6/18 has increased from 96.1 to $97 \%[1,5]$. Implementation of quality control by monitoring outcome measurements and providing interventions as needed is important, but this should be implemented without requiring excessive manpower, which would divert resources from healthcare delivery. Another utility in eye care planning is the OneHealth Tool, a program developed by the United Nations (UN) InterAgency Working Group on Costing for use in national healthcare planning [1]. It has been used in over 40 countries to date. OneHealth Tool will be adding eye care to its healthcare planning interventions in 2020 and we look forward to seeing its effectiveness in assisting eye care planning particularly in the setting of detecting and intervening early in cataract blindness.

\section{Refractive Error}

Far and away, the largest causes of vision impairment remain to be refractive error and presbyopia. The burden of myopia and presbyopia is increasing and unequally borne by lower socioeconomic status countries [6,7•]. An estimated 123.7 million people have vision impairment or blindness due to uncorrected refractive error and another 826 million have unaddressed presbyopia [1]. Living in a rural area or underdeveloped region and increasing age are associated with decreased likelihood of adequate optical correction [6]. Globally, potential productivity lost due to visual impairment attributed to myopia (estimated $\$ 244$ billion) greatly exceeds the cost of correcting myopia [6]. Worldwide, East Asia bears the greatest burden of myopia [6,7•]. Although increased screen time and near vision work have been suggested to correlate with myopia $[8,9]$, a new systematic review demonstrated mixed results and did not establish a clear causal relationship between screen time and increased myopia [10]. The 2-year results of a 5-year study of the low-concentration atropine for myopia progression study showed that atropine $0.05 \%$ (compared with atropine $0.025 \%, 0.01 \%$, and placebo) is the most effective dose for slowing myopia progression and axial elongation in children 4-12 years of age [11]. Public health efforts in the future need to address refractive error and reduce spectacle procurement to improve this large and growing cause of visual impairment.

\section{Diabetes}

Globally, vision impairment and blindness from diabetic retinopathy have increased over the decades from about 2.3 million visually impaired and 650,000 blind in 1990 to 3.7 million visually impaired and 834,000 blind in 2010 [12]. As the global burden of diabetes grows, these numbers are expected to increase, as much of the growth in diabetes rates are occurring in middle- and low-income countries. By 2045, an estimated 629 million people worldwide are expected to have diabetes (75\% in low- and middle-income countries) and about one-third of them are expected to have diabetic retinopathy [13]. Diabetic retinopathy is the leading cause of blindness in working age adults (age 20-65 years old). In Africa, the prevalence of diabetic retinopathy is about $15 \%$ of diabetics [14]. The presence and progression of diabetic retinopathy in African populations have been reported to be up to 5 times higher than in European populations and there are few retina specialists to provide management [15]. 
Artificial intelligence (AI) and deep learning have been proposed as solutions to the growing problem of visual impairment and blindness due to diabetic retinopathy. While most studies on $\mathrm{AI}$ and diabetic retinopathy have been conducted in high-income countries, a promising new development in 2019 and 2020 is the publication of multiple studies addressing the use of $\mathrm{AI}$ in low- and middle-income countries. The first AI pilot programs in Africa were published in 2015, using retrospective Kenyan patient images from the Nakuru Eye Study Cohort [16, 17]. In 2019, data from 5 urban centers in the Copperbelt Province of Zambia was published. This prospective study used AI to screen 1574 patients for diabetic retinopathy (i.e., referable diabetic retinopathy, visionthreatening diabetic retinopathy, and diabetic macular edema). Their sensitivity of detecting vision-threatening diabetic retinopathy or macular edema was $99.42 \%$ and $97.19 \%$, respectively. Rarely were critical cases missed by their AI system. In all cases, grading by the AI system was similar to human graders [18]. A key finding of this study was that the AI system was able to identify disease in an African cohort with high sensitivity and specificity even though the system was trained on patients of Asian origin from the Singapore Integrated Diabetic Retinopathy Program. A limitation of this study was the use of a high-complexity system (i.e., retina cameras and cloud-based telecommunications network). Systems that incorporate AI into retinal cameras may have an advantage in the developing world by eliminating the need for internet access. Another concern is that, once an image was identified as qualifying for a referral, there must be an eye care provider available who the patient can access. This can be difficult because a much lower ratio of ophthalmologists per diabetic retinopathy patients exists in sub-Saharan Africa compared with that in other regions of the world [19].

In circumstances where cloud-based and high network resource-dependent systems may not be possible due to lack of consistent electrical power or internet access, simpler technology may be indicated for screening. A prospective study based in Mumbai, India, used AI to examine Remidio smartphone-based, nonmydriatic images for diabetic retinopathy screening. In this study of 253 participants, this system detected referable diabetic retinopathy (i.e., diabetic retinopathy worse than mild, with or without macular edema) with a sensitivity of $100 \%$ and specificity of $88.4 \%$, and any form of diabetic retinopathy with a sensitivity of $85.2 \%$ and specificity of $92.0 \%$ [20]. Population-based screening programs like the UK program have reduced blindness due to diabetic retinopathy, but they require highly trained retinal graders and complex equipment. An advancement of the Mumbai study was the use of an off-line automated analysis algorithm rather than a processor and internet-intensive deep learning and AI networks, which may be difficult to access in the developing world. Two other data sets of patients have been published using the Remidio system in Bangalore and Chennai. In
Bangalore, among 297 patients screened, the sensitivity and specificity for detecting referable diabetic retinopathy were $98.84 \%$ and $86.73 \%$, respectively [21]. In Chennai, among 301 patients screened, the sensitivity and specificity for detecting any retinopathy was $95.8 \%$ and $80.2 \%$, respectively [22]. Of note, when the AI system gave a false negative for diabetic retinopathy, the patient often did have other fundus lesions that were worthy of referral (e.g., central and branch retinal vein occlusions, drusen), thus would have been appropriately referred to an ophthalmologist. In Thailand, using a Google-based AI system, a retrospective clinical validation study of 7517 patients with diabetes demonstrated a sensitivity of $96.8 \%$ and specificity of $95.6 \%$ for detecting moderate or worse non-proliferative diabetic retinopathy. In the Thailand study, the AI system was better or equal to regional graders for severe or worse non-proliferative diabetic retinopathy [23].

An excellent review of $\mathrm{AI}$ and telemedicine describes the experiences in many countries that are just beginning to implement the use of $\mathrm{AI}$ in screening for diabetic retinopathy, which includes country-specific reviews of the application of AI for diabetic retinopathy screening in China, the USA, the UK, Thailand, Australia, Singapore, and the African Continent [24]. In 2020, a review discussed current AI systems (e.g., IDx-DR (IDx Technologies Inc., Coralville, IA), RetmarkerDR or Retmarker Screening (RETMARKER S.A., Coimbra, Portugal), Singapore SERI-NUS (Singapore Eye Research Institute - National University of Singapore, Singapore), the Bosch DR algorithm (Robert Bosch GmbH, Bangalore, India), RetinaLyze ${ }^{\circledR}$ (RetinaLyze system A/S, Hellerup, Denmark)) [25]. The United States Food and Drug Administration has approved the IDx-DR AI system for detecting diabetic retinopathy levels more severe than mild and without the requirement for further interpretation by a clinician $[26,27]$.

Proponents of AI screening programs report the potential benefits in reducing reliance on manual work and savings in healthcare costs and resources compared with in-person screenings performed by an eye care provider. In particular, the cost and time of producing retina-trained ophthalmologists are prohibitive. Reduction of the need for specialists to screen diabetics may allow specialists to efficiently provide treatment to patients.

\section{Glaucoma}

Globally, the number of people with vision impairment and blindness due to glaucoma is about 6.9 million persons [1]. Treatment and diagnosis of glaucoma are often marred in lowresource environments by failure of early diagnosis, medication procurement, limited minimally invasive surgical options, and access to glaucoma providers. Artificial intelligence and 
deep learning algorithms have emerged as a potential powerful screening tool in the field of glaucoma. One study in the USA found that the detection of glaucoma from fundus images using AI was equivalent to or better than human graders [28]. Other studies are currently ongoing to apply deep learning algorithms in retinal nerve fiber layer OCTs in the diagnosis of glaucoma. To our knowledge, a prospective study of glaucoma detection via AI has yet to be published from the setting of the developing world, though it is foreseeable that a low-cost implantation of this technology may aid eye care professionals in the early diagnosis and treatment of glaucoma (Fig. 1a).

\section{Trachoma}

Chlamydia trachomatis is the leading infectious cause of blindness worldwide. Children are the main reservoir of
C. trachomatis, which is spread via ocular and nasal secretions. Blindness is the result of corneal scarring due to trichiasis from repeated infections from $C$. trachomatis leading to conjunctival scarring of the eyelid (Fig. 1b).

The WHO uses a "SAFE" (Surgery, Antibiotics, Face cleanliness, Environmental improvement) strategy for trachoma elimination from endemic areas. A 2019 WHO report states that, from 2012 to 2019, the number of districts where the $\mathrm{A}, \mathrm{F}$, and $\mathrm{E}$ components of SAFE are lacking has decreased from 1517 million to 142 million and the number of people with trachoma has fallen from 7.6 to 2.5 million [1]. Eight countries have been validated as having eliminated trachoma as a public health problem: Cambodia, Ghana, the Islamic Republic of Iran, Lao People's Democratic Republic, Mexico, Morocco, Nepal, and Oman. Other countries have achieved elimination targets: China, The Gambia, Iraq, Myanmar, and Togo. A recent study out of Southern Ethiopia confirmed lack of face washing, soap, and access to

Fig. 1 a Buphthalmos in primary congenital glaucoma in a 10 -yearold male. b Trachomatous trichiasis, superior and inferior entropion associated with corneal opacity. Courtesy of Ricardo Morschbacher MD PhD, Professor of UFCSPA, Brazil
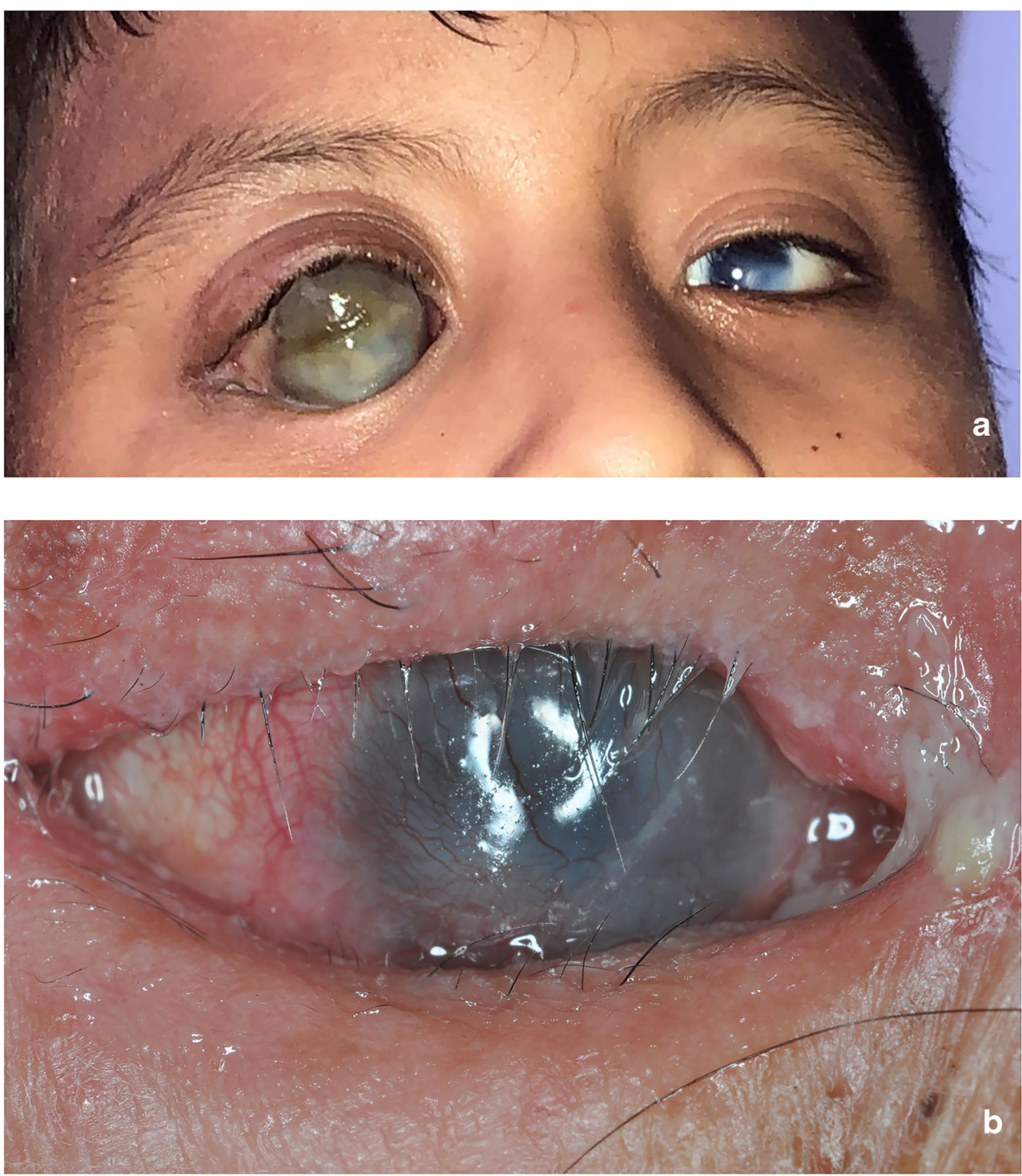
latrines was associated with active trachoma and face washing and environmental cleanliness were factors in trachoma prevention [29].

Antibiotics, a key component of the SAFE strategy, has been a hot topic over the last couple of years as results of the MORDOR (Macrolides Oraux pour Réduire les Décès avec un Oeil sur la Résistance) Trial have been published [30]. In particular, they investigated mass azithromycin distribution mechanisms and its effects on trachoma and childhood mortality. The MORDOR Trial, conducted in Malawi, Niger, and Tanzania, showed for the first time in a randomizedcontrolled clinical trial that mass administration for azithromycin reduced child mortality in sub-Saharan Africa. The primary endpoint, mortality in children aged 1 to 59 months, was reduced in all locations, especially in Niger (18\% mortality reduction). In this study, 190,238 children were monitored, and azithromycin and placebo were given two times a year over 2 years for a total of four doses. Mortality was $13.5 \%$ lower in the azithromycin group and this effect was greatest in Niger and in children 1-5 months of age [30]. This groundbreaking randomized-controlled clinical trial has led to more studies investigating the mechanism of mass azithromycin on both reducing trachoma and childhood mortality [31].

The timing, frequency of administration, discontinuation strategies, and effects of mass azithromycin administration on trachoma were reported in several trials. In Keenan et al. [32], the longitudinal effect of discontinuing a 4-year period of twice yearly mass distribution of azithromycin was that ocular chlamydia prevalence nearly doubled in communities where azithromycin was discontinued versus communities where it was continued. In the Amhara region of Ethiopia, an assessment of the progress of SAFE strategies was undertaken. The trachoma program began in Amhara in 2001 and expanded to all 152 districts between 2007 and 2010. During this study, from 2010 to 2015, this hyperendemic region was the first region in Ethiopia to complete trachoma impact surveys (conducted 7 to 9 months after last mass drug administration) in every district. Despite uptake of SAFE strategies and reductions in active trachoma (i.e., trachoma follicular (TF) infection and intense trachomatous inflammation (TI)), across the region, $37 \%$ of districts remained hyperendemic and only 9 districts out of 152 had prevalence of $\mathrm{TF}<5 \%$ in children aged 1-9 years, the trachoma elimination threshold [33]. These data suggest that 3-5-year annual drug administration programs may not be enough to reach trachoma elimination levels and perhaps 7-10-year programs in hyperendemic regions may be more appropriate [34].

Azithromycin administration in the Gambia and in Senegal led to decreased active trachoma (i.e., TF and TI), but did not decrease the prevalence of ocular $C$. trachomatis infection by Amplicor testing 1 year after a single round of treatment in children aged 1-9 years [35]. This study was unable to determine why there was poor correlation between active trachoma as graded by an ophthalmic nurse and $C$. trachomatis infection. Other studies have found a correlation between decreased clinical findings and decreased infection as determined by PCR testing at 3 and 6 months after a single administration of azithromycin; however, the decrease in inflammatory gene expression had returned to near pre-treatment levels at 6 months [36].

While current recommendations for communities to qualify for mass antibiotic treatment are based on the prevalence of TF in 1-9-year-old children [33], one study introduced a new term, "trachomatous papillary inflammation (TP)," which is an expansion of TI (defined as P3, severe papillary hypertrophy), including both P2 (moderate papillary hypertrophy) and P3. Based on their findings, they suggest that TP be added as a measure in trachoma control programs as an indication of the future risk of developing trichiasis [37]. This 4-year longitudinal cohort study, carried out in northern Tanzania to explore the relationship between $C$. trachomatis infection, clinical inflammation, and scarring development in children 6-10 year of age, found in multivariate analysis that scarring progression was strongly associated with increasing episodes of TP, and not associated with $C$. trachomatis infection or follicular trachomatous inflammation [37].

Currently, there are two major ongoing studies in treatments for trachoma. In 2019, Sie et al. published the study protocol for the Community Health with Azithromycin Trial (CHAT) which aims to assess child mortality in Burkina Faso with early azithromycin intervention in children between the 5 th and 12th weeks of life and to study the efficacy of twice annual azithromycin distribution on children under 5 years of age. The study began in August 2019 and plans to record macrolide resistance, serological markers of pathogen exposure, and child growth markers. Completion is expected in 2024 [38]. A second study, the Maximizing Trachoma Surgery Success (MTSS) trial, began in April 2017 to study trachomatous trichiasis surgery techniques. Enrollment is planned for a total of 6900 first-time trachoma surgery patients in Ethiopia and will follow them for 12 months after being randomized to one of the following surgical interventions: bilamellar tarsal rotation at $3 \mathrm{~mm}$ or at $5 \mathrm{~mm}$ from the lid margin, and posterior lamellar tarsal rotation (PLTR or Trabut procedure). Study completion is expected in 2021 [39].

\section{Coronavirus 2019}

At the time of this review, manuscripts are just starting to be published regarding the coronavirus 2019 (COVID-19) pandemic. With regard to eye care, some trends are beginning to emerge, including (1) the possibility of eye-related transmission of emerging infectious diseases [40]; (2) the presence of eye findings and disease in novel infectious disease such as 
COVID-19 [41, 42]; and (3) the potential for patients, ophthalmologists, and other eye care providers to be infected with these diseases through clinical and surgical eye care [43-45]. As the COVID-19 pandemic continues to spread, patient and provider infection control strategies will need to be developed and refined. Also, in the future, as new infectious diseases emerge, protocols planned and put in place now may help prevent future infection of patients and providers and the spread of disease through patient interactions.

\section{Retinopathy of Prematurity}

There is currently a "third epidemic" of blindness due to retinopathy of prematurity (ROP). The first epidemic of ROP occurred in the 1940s-1950s in industrialized countries due to primarily unmonitored supplemental oxygen, the second epidemic began in the 1970s in industrialized countries due to increased survival rates of extremely premature infants, and the third epidemic began in the mid-1990s in low- and middleincome countries (i.e., initially in Eastern Europe and Latin America, spreading to East and South Asia, and now in subSaharan Africa) due to both high rates of preterm birth and varying levels of neonatal care in these countries [46, 47]. In 2010 , it was estimated that, of the approximately 15 million premature births globally, $60 \%$ occur in south Asia and subSaharan Africa [48]. While appropriate screening and timely treatment have been shown to reduce the risk of blindness due to ROP, there are many obstacles to achieving this, especially in low- and middle-income countries. In many parts of the world, at-risk infants are not being screened and treated for ROP due to lack of country- or region-specific guidelines, not having enough ophthalmologists trained in how to screen and/ or treat ROP, and limited resources (e.g., availability of laser for treatment, family's ability to follow-up following hospital discharge) [49]. While ROP screening guidelines have been suggested in some Asian countries, many countries lack trained ophthalmologists to perform laser retinal ablation and pediatric retina surgery $[49,50]$. Sub-Saharan Africa collaborative working groups comprised of ophthalmologists and neonatologists have resulted in the development of national screening guidelines in South Africa and Kenya, and in a national screening protocol in Nigeria (serving as a precursor to developing national screening criteria) [47]. These advancements in ROP screening have been the result of committed local leaders, the aforementioned collaborative working groups, support from professional societies, and input from external experts [47]. A review of the literature found that, as of May 20, 2018, only 6 of 54 recognized sovereign African states had published data on ROP and the authors felt that, based on the rate of these publications, ROP seems to be emerging across the African continent [51•]. To increase ROP screening standards and reduce ROP-related blindness worldwide, countries need to establish realistic and countryspecific screening guidelines; to increase education, training, and collaboration between ophthalmologists and neonatologists; and to utilize other healthcare professionals in ROP screening (e.g., explore cost-effective telemedicine approaches to ROP screening) [52].

\section{Retinoblastoma}

It is known that the early diagnosis of retinoblastoma $(\mathrm{RB})$ can be both vision- and life-saving. The Global RB Study Group carried out a cross-sectional study of all new RB patients diagnosed in 2017 from 278 RB treatment centers from 153 countries, which was felt to have included $>50 \%$ of all new $\mathrm{RB}$ cases that year [53*0]. Approximately $85 \%$ of new cases were from low- or middle-income countries [53・•]. This study found that lower national income was associated with older age at presentation, higher proportion of more advanced disease, and a smaller proportion of family history of RB, the latter theorized due to fewer children with familial history of RB to survive to childbearing age [53••]. Interestingly, this study found that both lower national income level and older age at presentation were independently associated with advanced disease $[53 \cdot \bullet]$. Overall, the most common indications for referral were leukocoria (62.8\%), strabismus (10.2\%), and proptosis $(7.4 \%)[53 \cdot \bullet]$. Because RB is a curable disease, intervention at the national and international levels is merited and supported by the World Health Organization Global Initiative for Childhood Cancer in their efforts to raise survival for key childhood cancers.

\section{Conclusion}

As our world becomes more interconnected through globalization, barriers that once existed between eye care professionals have been upended. This has allowed for greater opportunities to collaborate with eye professionals and systems around the world to work towards the common goal of characterizing and reducing preventable visual impairment. Global ophthalmology is a branch of ophthalmology concerned with the influence of historical, environmental, cultural, political, and social conditions on eye health and disease epidemiology in all parts of the world. By learning and appreciating these factors, we can develop effective, data-driven methodologies to decrease visual impairment and blindness in any resource setting. This review describes the magnitude of the problems that global ophthalmology aims to address and outlines effective models that are currently being studied. To achieve our shared goal of eliminating preventable visual impairment in the near future, further studies and models need to be funded and constructed. 
Acknowledgments Dr. Raquel Goldhardt and Current Ophthalmology Reports would like to thank Dr. Cristos Ifantides, MD, for the careful review of this manuscript.

Funding Information NIH Center Core Grant P30EY014801 and Research to Prevent Blindness Unrestricted Grant.

\section{Compliance with Ethical Standards}

Conflict of Interest Lloyd B. Williams, S. Grace Prakalapakorn, and Zubair Ansari each declare no potential conflicts of interest.

Raquel Goldhardt is a section editor for Current Ophthalmology Reports.

Human and Animal Rights and Informed Consent This article does not contain any studies with human or animal subjects performed by any of the authors.

\section{References}

Papers of particular interest, published recently, have been highlighted as:

- Of importance

•- Of major importance

1. World Report on Vision. 2019, World Health Organization

2. Khanna RSS, Sabherwal S, Sil A, Gowth M, Dole K, Kuyyadiyil S, et al. Primary eye care in India - the vision center model. Indian J Ophthalmol. 2020;68(2):333-9.

3. Muhammed N, et al. Impact of a 10-year eye care program in Sokoto, Nigeria: changing pattern of prevalence and causes of blindness and visual impairment. Middle East Afr J Ophthalmol. 2019;26(2):101-6.

4. Mehta MC, Narayanan R, Thomas Aretz H, Khanna R, Rao GN. The L V Prasad Eye Institute: a comprehensive case study of excellent and equitable eye care. Healthc (Amst). 2020;8(1):100408.

5. Salowi MA, Choong YF, Goh PP, Ismail M, Lim TO. CUSUM: a dynamic tool for monitoring competency in cataract surgery performance. Br J Ophthalmol. 2010;94:445-9.

6. Naidoo KS, Fricke TR, Frick KD, Jong M, Naduvilath TJ, Resnikoff S, et al. Potential lost productivity resulting from the global burden of myopia. Ophthalmology. 2019;126:338-46.

7. Wang Y, et al. Socio-economic disparity in global burden of near vision loss: an analysis for 2017 with time trends since 1990. Acta Ophthalmol. 2020;98:e138-43.

8. Ku P-W, Steptoe A, Lai YJ, Hu HY, Chu D, Yen YF, et al. The associations between near visual activity and incident myopia in children: a nationwide 4-year follow-up study. Ophthalmology. 2019;126(2):214-20.

9. Huang H-M, Chang DS-T, Wu P-C. The association between near work activities and myopia in children - a systematic review and meta-analysis. PLoS One. 2015;10(10):e1-15.

10. Lanca $\mathrm{C}$, Saw S-M. The association between digital screen time and myopia: a systematic review. Ophthalmic Physiol Opt. 2019;40: 216-29.

11. Yam JC, Li FF, Zhang X, et al. Two-year clinical trial of the LowConcentration Atropine for Myopia Progression (LAMP) Study: phase 2 report [published online ahead of print, 2019 Dec 21]. Ophthalmology. 2019;:S0161-6420(19):32356-5.

12. Leasher J, Bourne RR, Flaxman SR, Jonas JB, Keeffe J, Naidoo K, et al. Global estimates on the number of people blind or visually impaired by diabetic retinopathy: a meta-analysis from 1990 to 2010. Diabetes Care. 2016;39(9):1643-9.

13. Mohan V, Khunti K, Chan SP, Filho FF, Tran NQ, Ramaiya K, et al. Management of type 2 diabetes in developing countries: balancing optimal glycaemic control and outcomes with affordability and accessibility to treatment. Diabetes Ther. 2019;11(1):15-35.

14. Ekoru K, Doumatey A, Bentley AR, Chen G, Zhou J, Shriner D, et al. Type 2 diabetes complications and comorbidity in subSaharan Africans. EClinicalMedicine. 2019;16:30-41.

15. Burgess $P$, et al. Incidence and progression of diabetic retinopathy in sub-Saharan Africa: a five-year cohort study. PLoS One. 2017;12(8):e0181359.

16. Bastawrous A, et al. The Nakuru Eye Disease Cohort Study: methodology and rationale. BMC Ophthalmol. 2014;14:e2-10.

17. Hansen MB, et al. Results of automated retinal image analysis for detection of diabetic retinopathy from the Nakuru study, Kenya. PLoS One. 2015;10(10):e1-9.

18. Bellemo V, Lim ZW, Lim G, Nguyen QD, Xie Y, Yip MYT, et al. Artificial intelligence using deep learning to screen for referable and vision-threatening diabetic retinopathy in Africa: a clinical validation study. Lancet Digit Health. 2019;1:e35-44.

19. Teo ZL, et al. Do we have enough ophthalmologists to manage vision-threatening diabetic retinopathy? A global perspective [published online ahead of print, 2020 Jan 28]. Eye (Lond). 2020; https://doi.org/10.1038/s41433-020-0776-5.

20. Natarajan S, Jain A, Krishnan R, Rogye A, Sivaprasad S. Diagnostic accuracy of community-based diabetic retinopathy screening with an offline artificial intelligence system on a smartphone. JAMA Ophthalmol. 2019;137(10):1182-8.

21. Sosale B, et al. Medios - an offline, smartphone-based artificial intelligence algorithm for the diagnosis of diabetic retinopathy. Indian J Ophthalmol. 2020;68(2):391-5.

22. Rajalakshmi R, Subashini R, Anjana RM, Mohan V. Automated diabetic retinopathy detection in smartphone-based fundus photography using artificial intelligence. Eye (Lond). 2018;32:1138-44.

23. Raumviboonsuk $P$, et al. Deep learning versus human graders for classifying diabetic retinopathy severity in a nationwide screening program. NPJ Digit Med. 2019;2(25):1-9.

24. Bellemo V, et al. Artificial intelligence screening for diabetic retinopathy: the real-world emerging application. Curr Diab Rep. 2019;19(72):1189-93.

25. Grzybowski A, Brona P, Lim G, Ruamviboonsuk P, Tan GSW, Abramoff M, et al. Artificial intelligence for diabetic retinopathy screening: a review. Eye (Lond). 2020;34:451-60.

26. Abràmoff $\mathrm{MD}$, et al. Improved automated detection of diabetic retinopathy on a publicly available dataset through integration of deep learning. Invest Ophthalmol Vis Sci. 2016;57(13):5200-6.

27. Wong TY, Sabanayagam C. Strategies to tackle the global burden of diabetic retinopathy: from epidemiology to artificial intelligence. Ophthalmologica. 2020;243:9-20.

28. Jammal AA, et al. Human versus machine: comparing a deep learning algorithm to human gradings for detecting glaucoma on fundus photographs. Am J Ophthalmol. 2020;211:123-31.

29. WoldeKidan E, et al. Prevalence of active trachoma and associated factors among children aged 1 to 9 years in rural communities of Lemo District, southern Ethiopia: community based cross sectional study. BMC Infect Dis. 2019;19(1):e1-8.

30. Keenan JD, Bailey RL, West SK, Arzika AM, Hart J, Weaver J, et al. Azithromycin to reduce childhood mortality in sub-Saharan Africa. N Engl J Med. 2018;378:1583-92.

31. Keenan JD, Arzika AM, Maliki R, Elh Adamou S, Ibrahim F, Kiemago M, et al. Cause-specific mortality of children younger than 5 years in communities receiving biannual mass azithromycin treatment in Niger: verbal autopsy results from a clusterrandomized controlled trial. Lancet. 2020;8:e288-95. 
32. Keenan JD, et al. Mass azithromycin distribution for hyperendemic trachoma following a cluster-randomized trial: a continuation study of randomly reassigned subclusters (TANA II). PLoS Med. 2018;15(8):1-17.

33. Trachoma control: a guide for programme managers. World Health Organization. 2006.

34. Stewart AEP, Zerihun M, Gessese D, Melak B, Sata E, Nute AW, et al. Progress to eliminate trachoma as a public health problem in Amhara National Regional State, Ethiopia: results of 152 population based surveys. Am J Trop Med Hyg. 2019;101(6):1286-95.

35. Holland $\mathrm{MH}$, et al. Impact of a single round of mass drug administration with azithromycin on active trachoma and ocular chlamydia trachomatis prevalence and circulating strains in the Gambia and Senegal. Parasit Vectors. 2019;12(1):497.

36. Ramadhani AM, et al. Ocular immune responses, chlamydia trachomatis infection and clinical signs of trachoma before and after azithromycin mass drug administration in a treatment naive trachoma-endemic Tanzanian community. PLoS Negl Trop Dis. 2019;13(7):e1-18.

37. Ramadhani AM, et al. Progression of scarring trachoma in Tanzanian children: a four-year cohort study. PLoS Negl Trop Dis. 2019;13(8):e1-16.

38. Sie A, et al. A double-masked placebo-controlled trial of azithromycin to prevent child mortality in Burkina Faso, West Africa: Community Health with Azithromycin Trial (CHAT) study protocol. Trials. 2019;20:675-86.

39. Bayissasse B, et al. Maximising Trichiasis Surgery Success (MTSS) trial: rationale and design of a randomized controlled trial to improve trachomatous trichiasis surgical outcomes. BMJ Open. 2020;10:e1-8.

40. Qing H, et al. The possibility of COVID-19 transmission from eye to nose. Acta Ophthalmol. 2020;98(3):e388.

41. Liang $\mathrm{L}, \mathrm{Wu} \mathrm{P}$. There may be virus in conjunctival secretion of patients with COVID-19. Acta Ophthalmol. 2020;98(3):223.

42. Wu P, et al. Characteristics of ocular findings of patients with coronavirus 2019 (COVID-19) in Hubei Province, China. JAMA Ophthalmol. 2020;138(5):575-8.

43. Jørstad ØK, Moe MC, Eriksen K, Petrovski G, Bragadóttir R. Coronavirus disease 2019 (COVID-19) outbreak at the Department of Ophthalmology, Oslo University Hospital, Norway. Acta Ophthalmol. 2020;98(3):e388-9.

44. Lai THT, Tang EWH, Chau SKY, Fung KSC, Li KKW. Stepping up infection control measures in ophthalmology during the novel coronavirus outbreak: an experience from Hong Kong. Graefes Arch Clin Exp Ophthalmol. 2020;258(5):1049-55.

45. Wan KH, Huang SS, Young AL, Lam DSC. Precautionary measures needed for ophthalmologists during pandemic of the coronavirus disease of 2019 (Covid-19). Acta Ophthalmol. 2020;98(3): 221-2.

46. Gilbert C. Retinopathy of prematurity: a global perspective of the epidemics, population of babies at risk and implications for control. Early Hum Dev. 2008;84(2):77-82.

47. Gilbert C, Malik ANJ, Nahar N, Das SK, Visser L, Sitati S, et al. Epidemiology of ROP update - Africa is the new frontier. Semin Perinatol. 2019;43(6):317-22.

48. Blencowe H, Cousens S, Oestergaard MZ, Chou D, Moller AB, Narwal R, et al. National, regional, and worldwide estimates of preterm birth rates in the year 2010 with time trends since 1990 for selected countries: a systematic analysis and implications. Lancet. 2012;379(9832):2162-72.

49. Sen P, Wu WC, Chandra P, Vinekar A, Manchegowda PT, Bhende P. Retinopathy of prematurity treatment: Asian perspectives. Eye (Lond). 2020;34(4):632-42.

50. Chan DFF, Herrera-Arroyo MM. Anatomic outcomes of laser indirect ophthalmoscopy for retinopathy of prematurity in a tertiary referral center in the Philippines. BMC Res Notes. 2019;12(1):263.

51. Wang D, Duke R, Chan RP, Campbell JP. Retinopathy of prematurity in Africa: a systematic review. Ophthalmic Epidemiol 2019;26:223-30. This systematic review summarizes the published literature on ROP from African nations. The results of this review can help future planning of ROP efforts.

52. Adams GGW. ROP in Asia. Eye (Lond). 2020;34(4):607-8.

53.• Global Retinoblastoma Study Group, Fabian ID, Abdallah E, et al. Global retinoblastoma presentation and analysis by national income level. JAMA Oncol. 2020;6(5):1-12; This cross-sectional study of all new RB patients diagnosed across the world in 2017 , felt to include $>50 \%$ of new RB that year, allowed investigation of associations between clinical variables and national income level and risk factors for advanced disease at diagnosis. The results of this study can help efforts focused on improving RB diagnosis.

Publisher's Note Springer Nature remains neutral with regard to jurisdictional claims in published maps and institutional affiliations. 